\title{
Intranodal signal suppression in pelvic MR lymphography of prostate cancer patients: a quantitative comparison of ferumoxtran-10 and ferumoxytol
} \author{
Jelle O. Barentsz ${ }^{1}$, Henkjan J. Huisman ${ }^{1}$ \\ 1 Department of Radiology and Nuclear Medicine, Radboud umc, Nijmegen, The Netherlands \\ 2 Department of Radiology, Ziekenhuis Gelderse Vallei, Ede, The Netherlands \\ Corresponding Author: Oscar A. Debats \\ Email address: debats@gmail.com
}

Oscar A. Debats ${ }^{\text {Corresp., }}{ }^{1}$, Ansje S. Fortuin ${ }^{2}$, Hanneke J.M. Meijer ${ }^{1}$ ， Thomas Hambrock ${ }^{1}$, Geert J.S. Litjens ${ }^{1}$,

OBJECTIVES: The key to MR lymphography is suppression of T2* MR signal in normal lymph nodes, while retaining high signal in metastatic nodes. Our objective is to quantitatively compare the ability of ferumoxtran-10 and ferumoxytol to suppress the MR signal in normal pelvic lymph nodes. METHODS: In 2010, a set of consecutive patients who underwent intravenous MR Lymphography (MRL) were included. Signal suppression in normal lymph nodes in T2*-weighted images due to uptake of USPIO (Ultra-Small Superparamagnetic Particles of Iron Oxide) was quantified. Signal suppression by two USPIO contrast agents, ferumoxtran-10 and ferumoxytol was compared using Wilcoxon's signed rank test. RESULTS: Forty-four patients were included, of which all 44 had a ferumoxtran-10 MRL and 4 had additionally a ferumoxytol MRL. A total of 684 lymph nodes were identified in the images, of which 174 had been diagnosed as metastatic. USPIOinduced signal suppression in normal lymph nodes was significantly stronger in ferumoxtran-10 MRL than in ferumoxytol MRL $(p<0.005)$. CONCLUSIONS: T2* signal suppression in normal pelvic lymph nodes is significantly stronger with ferumoxtran-10 than with ferumoxytol, which may affect diagnostic accuracy. 
1 Title Page

3 Intranodal signal suppression in pelvic MR lymphography of prostate cancer

4 patients: a quantitative comparison of ferumoxtran-10 and ferumoxytol.

5 Oscar A. Debats ${ }^{1}$, Ansje S. Fortuin ${ }^{2}$, Hanneke J.M. Meijer ${ }^{1}$, Thomas Hambrock ${ }^{1}$, Geert J.S.

6 Litjens $^{1}$, Jelle O. Barentsz ${ }^{1}$, Henkan J. Huisman ${ }^{1}$

$8{ }^{1}$ Department of Radiology and Nuclear Medicine, Radboud University Medical Centre, 9 Nijmegen, The Netherlands

$10{ }^{2}$ Department of Radiology, Ziekenhuis Gelderse Vallei, Ede, The Netherlands

11

12 Corresponding Author:

13

14 Oscar A. Debats ${ }^{1}$

15 Kaaplandstraat 55 D, Nijmegen 6543 PC, The Netherlands

16 Email address: debats@gmail.com 


\section{Abstract}

\section{Objectives}

20 The key to MR lymphography is suppression of T2* MR signal in normal lymph nodes, while

21 retaining high signal in metastatic nodes. Our objective is to quantitatively compare the ability of

22 ferumoxtran-10 and ferumoxytol to suppress the MR signal in normal pelvic lymph nodes.

\section{Methods}

24 In 2010, a set of consecutive patients who underwent intravenous MR Lymphography (MRL)

25 were included. Signal suppression in normal lymph nodes in T2*-weighted images due to uptake

26 of USPIO (Ultra-Small Superparamagnetic Particles of Iron Oxide) was quantified. Signal

27 suppression by two USPIO contrast agents, ferumoxtran-10 and ferumoxytol was compared

28 using Wilcoxon's signed rank test.

\section{Results}

30 Forty-four patients were included, of which all 44 had a ferumoxtran-10 MRL and 4 had

31 additionally a ferumoxytol MRL. A total of 684 lymph nodes were identified in the images, of

32 which 174 had been diagnosed as metastatic. USPIO-induced signal suppression in normal

33 lymph nodes was significantly stronger in ferumoxtran-10 MRL than in ferumoxytol MRL

$34(\mathrm{p}<0.005)$. 


\section{Conclusions}

$36 \mathrm{~T} 2 *$ signal suppression in normal pelvic lymph nodes is significantly stronger with ferumoxtran-

3710 than with ferumoxytol, which may affect diagnostic accuracy. 


\section{Introduction}

40 Prostate cancer (PCa) is the most common type of cancer and the second leading cause of cancer

41 death in men [1]. The presence of lymph node metastases is a poor prognostic factor, reducing

42 treatment options. Conventional imaging modalities such as CT or MRI rely on size and shape

43 criteria to detect metastatic lymph nodes, resulting in poor sensitivity and specificity [2, 3].

44 Pelvic lymph node dissection (PLND) is currently regarded as the gold standard for lymph node

45 staging in PCa patients, but comes with increased costs and risk of morbidity [4, 5], and not all

46 lymph node metastases are found at routine PLND [6]. To select patients for PLND or elective

47 nodal irradiation, various nomograms $[7,8]$ and numerical formulae $[9,10]$ are used to predict

48 nodal involvement. However, these do not provide information on the number, size, and location

49 of metastatic nodes, which are important parameters for staging [11]. 11C-choline PET/CT has

50 been shown to be more accurate than CT and MR [12], but has limited sensitivity in the

51 substantial group of smaller lymph node metastases $<7 \mathrm{~mm}$ [13].

52 MR Lymphography (MRL) outperforms 11C-choline PET/CT by providing good accuracy for

53 lymph nodes well below $7 \mathrm{~mm}$. MRL uses a specific, Ultra-Small Superparamagnetic Particles

54 of Iron Oxide (USPIO) based contrast agent. USPIOs accumulate in macrophages in normal

55 lymphatic tissue, resulting in signal suppression on T2*-weighted MRI. Normal lymph nodes

56 become dark, and when fat-saturation is applied, fade into the background of the surrounding

57 dark fat. Thus, metastatic lymph nodes stand out with bright signal intensity (SI) [14]. MRL

58 properly performed and interpreted can provide a high negative predictive value (NPV) (95- 
59 99\%). A substantial amount of prostate cancer patients can thus be spared a PLND procedure and

60 the associated risk of morbidity.

61 Two MRL USPIO contrast agents can be currently used for humans. MRL using ferumoxtran-10

62 (Combidex ${ }^{\circledR}$, SPL Medical, Nijmegen, The Netherlands) is the only prospectively investigated

63 imaging modality for assessing metastatic involvement of pelvic lymph nodes, with sensitivities

64 up to $91 \%$, at $98 \%$ specificity [15]. Despite these encouraging results, ferumoxtran-10 has not

65 yet reached the market. Currently, ferumoxtran-10 is produced in The Netherlands by SPL

66 Medical B.V. under GMP (good manufacturing practice) conditions. The pharmacy of our

67 institution takes care of the supply of ferumoxtran-10 (for clinical routine and for clinical trials)

68 for its patients in accordance with the Dutch law.

69 The iron replacement drug ferumoxytol [16] (Feraheme ${ }^{\circledR}$, AMAG Pharmaceuticals Inc,

70 Cambridge, MA) has been proposed as a potential alternative MRL contrast agent. In 2007,

71 Harisinghani et al concluded that ferumoxytol MRL potentially identifies malignant lymph nodes

72 [17]. To the best of our knowledge however, no prospective studies have yet been performed to

73 validate the off-label use of this drug as an MRL contrast agent. Moreover, the FDA has issued a

74 warning considering its off-label use, and explicitly recommends intravenous administration by a

75 slow drip infusion: In the safety announcement, issued on 30 March 2015, which is available on

76 the website www.fda.gov, it is stated that all IV iron products carry a risk of potentially life-

77 threatening allergic reactions, and that a Boxed Warning had been added to the prescribing

78 instructions of ferumoxytol that describes these serious risks and specifies a number of 
79 recommendations. One of these recommendations is 'Only administer diluted Feraheme as an IV

80 infusion over a minimum of 15 minutes. Feraheme should not be given as an undiluted IV

81 injection.’

82 The purpose of this pilot study was to quantitatively compare ferumoxytol and ferumoxtran-10

83 for use in MRL. The outcome measure was signal suppression in normal lymph nodes, as this is

84 the basis for discriminating metastatic and normal ones.

\section{Materials and Methods}

86 This study retrospectively evaluated clinically obtained MRL data. The scientific use of

87 clinically obtained image data was approved by the Institutional Review Board. All patients

88 provided written informed consent for the use of the obtained data for research purposes.

89 Patient Selection

90 Between January and April 2010, a set of consecutive patients who had been referred to the

$91<$ BLINDED $>$ hospital for clinically indicated MRL were included in this retrospective study.

92 The inclusion criteria were: 1) a histologically confirmed prostate cancer with intermediate to

93 high risk for nodal metastases; 2) a ferumoxytol MRL and/or ferumoxtran-10 MRL performed

94 between January and April 2010; 3) successfully acquired 3D T1-weighted ("VIBE”) and 3D

95 T2*-weighted (“MEDIC”) sequences available.

96 MRL Protocol 
97 The MRL protocol was as follows. USPIO contrast was administered intravenously, 36 to 24

98 hours before the MRI was performed. Because of this time interval, only post-contrast images

99 were acquired. The same time interval was applied for both types of MRL. For ferumoxtran-10

100 MRL, this has been established as the optimal interval. For ferumoxytol MRL, this was

101 considered optimal by our expert readers based on visual inspection of a set of nine MRLs

102 acquired from three patients at different time intervals post injection. One patient was imaged at 103 day 0 (directly post injection), at day 1, and at day 2, and the other two were imaged at day 0 , 104 day 1 , and day 3.

105 Immediately before imaging, Buscopan (20 mg i.v. and 20 mg i.m.) and Glucagon (20 mg i.m.)

106 were administered to suppress bowel peristalsis. The dose of ferumoxtran-10 was 2.6 mg Fe per $107 \mathrm{~kg}$ body weight, conform earlier research [15]. The dose of ferumoxytol was $6.0 \mathrm{mg}$ Fe per kg

108 body weight. This is the maximum allowed dose, which was chosen to maximize the signal 109 suppression in the MEDIC images.

110 Imaging was performed using a 3.0 Tesla MR-scanner (Magnetom TrioTim; Siemens, Erlangen, 111 Germany). Scan parameters are listed in Table 1.

\section{Interpretation of MRL images}

113 The radiological diagnosis of the MRL examinations was established as the consensus reading

114 by two expert readers: an MD specially trained in reading MRL scans (<BLINDED>, 2 years of

115 MRL experience, >300 MRLs), and an abdominal radiologist (<BLINDED>, >10 years of MRL 
116 experience, >1000 MRLs). The T1-weighted sequences (see Table 1) were used for localization

117 and assessment of shape and size of the lymph nodes, and the iron-sensitive T2*-weighted

118 sequences were used to assess USPIO uptake, as described by Heesakkers et al [3].

\section{Quantitative MRL Analysis}

120 The contrast uptake in all detected lymph nodes was computed as follows. All lymph nodes

121 visible in the pelvic region were interactively segmented using the computer application Lymph

122 Node Task Card (Siemens, Malvern, PA). Segmentation was performed based on the T1-

123 weighted images, in which all lymph nodes (normal as well as metastatic ones) appear as

124 hypointense structures. From these three-dimensional segmentations, the volume of each lymph

125 node was recorded automatically.

126 As a measure of contrast uptake, relative SI was computed rather than absolute SI. Lymph node

127 assessment based on relative SI (local fat calibrated lymph node assessment) is more similar to

128 visual assessment of the MRL image by a radiologist: visual assessment is also based on a

129 comparison of the SI of a lymph node with the SI of the surrounding fat. Local fat calibration

130 compensates for coil profiles and other factors creating a spatially varying SI distortion in the

131 images. The fat calibration was implemented by manually segmenting a region of fatty tissue in

132 the direct neighborhood of each lymph node. The relative SI is calculated by subtraction of the

133 mean SI of the local fatty tissue region from the SI in the corresponding lymph node. 


\section{Statistical Analysis}

135 Relative SI in normal lymph nodes was compared using Wilcoxon’s signed rank test for paired 136 non-parametric data, using the statistical software package SPSS (version 20.0). P-values $<0.05$

137 were considered statistically significant. Box-and-whisker plots were constructed using the R

138 Environment for Statistical Computing.

\section{Results}

140 Forty-four patients fulfilled the inclusion criteria. All patients had prostate cancer staged as

141 Gleason Score 6 or higher. All underwent ferumoxtran-10 MRL, and four patients also

142 underwent ferumoxytol MRL. In all cases, ferumoxtran-10 MRL was performed first. The time

143 intervals between the two types of MRL for these four patients were as follows: 23, 130, 240,

144 and 241 days (0.8, 4.3, 7.9 and 7.9 months) respectively. Median prostate specific antigen (PSA)

145 and Gleason Score were 9.5 (range 0.01-954.0) and 7 (range 6-9), respectively. A total of 684

146 lymph nodes were identified in the MRL images, and 57 (8.3\%) of those belonged to patients

147 who received both types of MRL. All 684 nodes were found to be suitable for quantification of

148 signal suppression. The readers diagnosed 174 lymph nodes (25\%) as positive (i.e. metastatic).

149 Median size (volume) of the positive lymph nodes was $0.14 \mathrm{ml}$ (inter-quartile range (IQR) 0.043

$150-0.45)$ and median volume of the negative lymph nodes was $0.12 \mathrm{ml}($ IQR $0.045-0.30)$. A

151 histogram of lymph node sizes is shown in Figure 1. 
152 Visual evaluation of the MEDIC images demonstrated that in ferumoxtran-10 MRL the signal of

153 normal lymph nodes was markedly suppressed; thus they were generally as dark as the

154 surrounding (suppressed) fatty tissue, whereas metastatic nodes remained visible as bright

155 structures. With ferumoxytol, normal nodes also had suppressed signal but the suppression was

156 less than with ferumoxtran-10, and they remained brighter than the fatty tissue background, still

157 apparent as hyperintense structures (Figure 2).

158 The signal intensity box plot (Figure 3) shows that with ferumoxtran-10, the interquartile range

159 (IQR) of normal lymph node intensity had an overlap with the IQR of fatty tissue intensity.

160 However, with ferumoxytol, the IQR of normal lymph nodes did not overlap with the IQR of

161 fatty tissue.

162 The difference in relative SI of normal lymph nodes between the two types of MRL was

163 significant. Relative SI was on average 39.7 (95\% confidence interval (CI): [31.1, 48.3]) in

164 ferumoxytol MRL, and -2.1 (95\% CI: [-8.0, 3.8]) in ferumoxtran-10 MRL $(\mathrm{p}<0.005)$. 


\section{Discussion}

167 The results of this pilot study show, that relative SI in normal lymph nodes was significantly

168 higher in post-contrast ferumoxytol MRL than in ferumoxtran-10 MRL, both visually and

169 quantitatively $(\mathrm{p}<0.005)$. The MRL protocol used in this study included only post-contrast

170 imaging. However, as pre-contrast SI (whether absolute or relative) is not influenced, of course,

171 by the choice of contrast agent, pre-contrast SI would not differ between ferumoxytol MRL and

172 ferumoxtran-10 MRL, and any significant difference in relative SI refers to a difference in signal

173 suppression. In other words, the difference in relative SI found in our analysis implies that signal

174 suppression was weaker for ferumoxytol MRL than for ferumoxtran-10 MRL.

175 The underlying mechanism causing the difference in signal suppression in normal lymph nodes

176 is not known. Possibly it can be explained by the different coating of the particles, which may

177 lead to different uptake by macrophages, or different clearance. A limited number of studies have

178 been published comparing ferumoxtran-10 and ferumoxytol as MR contrast agent. Interestingly,

179 both agents appear suitable for detection of macrophages in atherosclerotic plaques [18], and for

180 detection of antigen-induced arthritis [19]. A possible explanation for the difference in behavior

181 when used for lymph node imaging may be the selective uptake of ferumoxtran-10, but not

182 ferumoxytol, by macrophages that migrate specifically to nodal tissue. This might be caused by

183 the different coatings of ferumoxtran-10, or by the different hydrodynamic diameter. In a recent

184 study comparing three USPIO’s in a porcine model, it was shown that differences in 
185 hydrodynamic diameter were associated with significant differences in lymphatic iron 186 accumulation [20].

187 The MR sequences used in this study have been validated in earlier research [3]. One might 188 argue that the lesser signal suppression in ferumoxytol MRL could be improved by optimizing 189 the sequences to make them more sensitive to USPIO. However, this would lead to an increase 190 of artifacts related to bowel peristalsis, which would deteriorate image quality.

191 The vast majority of the lymph nodes in our data set are of normal size (i.e. with short-axis $<10$ 192 diameter). As can be seen in Figure 1, the distribution of lymph node size does not differ 193 substantially between positive and negative lymph nodes. This is in accordance with the results 194 reported by Tiguert et al, who analyzed 980 prostatectomy patients and concluded that in 195 normal-sized lymph nodes, size did not correlate with the presence of metastasis [21].

196 This study has some limitations. In this pilot study, number of patients was limited: only four 197 patients received both types of MRL. However, by performing a quantitative analysis on a nodal 198 level in the same set of node-by-node compared lymph nodes we were able to perform a valid 199 analysis of the differences. A total of 684 nodes, of which 57 were imaged with both contrast 200 agents, were analyzed, and demonstrated a significant difference in intra-nodal signal 201 suppression between the two contrast agents. In future studies these results need to be confirmed 202 by including a larger number of patients with a ferumoxytol MRL. 
203 Another limitation is that the optimal time interval - 24 to 36 hours - between contrast

204 administration and imaging has only been investigated qualitatively for ferumoxytol MRL. As

205 described in the Methods section, this interval was considered optimal based on visual inspection

206 of a set of ferumoxytol MRLs acquired after different time intervals, but a quantitative

207 investigation is needed to create a firmer basis for this choice.

208 Radiologists who want to use ferumoxytol as an alternative to ferumoxtran-10 should be aware

209 that MRL with ferumoxytol as a contrast agent needs to be interpreted differently even with

210 identical MR sequences. In ferumoxtran-10 MRL, a lymph node that remains bright is highly

211 suspicious for metastasis, but this clear separation does not apply for ferumoxytol MRL. Thus

212 the distinction between normal and metastatic nodes is less straightforward. This is illustrated in

213 Figure 3, where the box plots of ferumoxytol-normal and ferumoxytol-metastatic overlap

214 substantially, which does not occur with ferumoxtran-10. Thus, using ferumoxytol may lead to

215 either many more false positives, or much lower sensitivity. Considering the already relatively

216 low PPV of 69\% of ferumoxtran-10 [3], a significant further decrease in PPV with ferumoxytol

217 may be problematic and lead to diagnostic inaccuracy or uncertainty. This is important because

218 ferumoxtran-10 is available on a limited scale, whereas ferumoxytol can be used off-label. Thus,

219 knowledge about the advantages and disadvantages of ferumoxytol is crucial.

220 In conclusion: USPIO-induced signal suppression in normal lymph nodes is significantly weaker

221 for ferumoxytol than for ferumoxtran-10, and therefore its discriminative performance is likely

222 to be lower. Therefore, the successful results reported in previous studies regarding ferumoxtran- 
22310 MRL cannot be extrapolated to ferumoxytol MRL. Ferumoxytol MRL, when used in an off224 label mode as a replacement for ferumoxtran-10 MRL, is likely to result in more false positives, 225 and should not be used in clinical practice for the diagnosis of metastatic lymph nodes without 226 further scientific validation. 
228 [1] R. L. Siegel, K. D. Miller, and A. Jemal, “Cancer statistics, 2015,” CA: A Cancer Journal 229 for Clinicians, vol. 65, pp. 5-29, 2015.

230 [2] A. M. Hövels, R. A. M. Heesakkers, E. M. Adang, G. J. Jager, S. Strum, Y. L.

231 Hoogeveen, J. L. Severens, and J. O. Barentsz, “The diagnostic accuracy of CT and MRI in the 232 staging of pelvic lymph nodes in patients with prostate cancer: a meta-analysis,” Clinical 233 Radiology, vol. 63, pp. 387-395, 2008.

234 [3] R. A. M. Heesakkers, A. M. Hövels, G. J. Jager, H. C. M. van den Bosch, J. A. Witjes, 235 H. P. J. Raat, J. L. Severens, E. M. M. Adang, C. Hulsbergen van der Kaa, J. J. Fütterer, and 236 J. Barentsz, "MRI with a lymph-node-specific contrast agent as an alternative to CT scan and 237 lymph-node dissection in patients with prostate cancer: a prospective multicohort study,” Lancet 238 Oncology, vol. 9, pp. 850-856, 2008.

239 [4] A. Heidenreich, J. Bellmunt, M. Bolla, S. Joniau, M. Mason, V. Matveev, N. Mottet, H.240 P. Schmid, T. van der Kwast, T. Wiegel, F. Zattoni, and E. A. o. U. , "EAU guidelines on 241 prostate cancer. part 1: screening, diagnosis, and treatment of clinically localised disease,” 242 European Urology, vol. 59, pp. 61-71, 2011.

243 [5] S. Loeb, A. W. Partin, and E. M. Schaeffer, "Complications of pelvic lymphadenectomy: 244 do the risks outweigh the benefits?,” Reviews in Urology, vol. 12, pp. 20-24, 2010. 
245 [6] R. A. M. Heesakkers, G. J. Jager, A. M. Hövels, B. de Hoop, H. C. M. van den Bosch,

246 F. Raat, J. A. Witjes, P. F. A. Mulders, C. Hulsbergen van der Kaa, and J. O. Barentsz, "Prostate

247 cancer: detection of lymph node metastases outside the routine surgical area with ferumoxtran-

248 10-enhanced MR imaging,” Radiology, vol. 251, pp. 408-414, 2009.

249 [7] A. W. Partin, J. Yoo, H. B. Carter, J. D. Pearson, D. W. Chan, J. I. Epstein, and P. C.

250 Walsh, “The use of prostate specific antigen, clinical stage and Gleason score to predict

251 pathological stage in men with localized prostate cancer,” Journal of Urology, vol. 150, pp. 110-

$252114,1993$.

253 [8] A. W. Partin, M. W. Kattan, E. N. Subong, P. C. Walsh, K. J. Wojno, J. E. Oesterling,

254 P. T. Scardino, and J. D. Pearson, “Combination of prostate-specific antigen, clinical stage, and

255 Gleason score to predict pathological stage of localized prostate cancer. A multi-institutional

256 update,” Journal of the American Medical Association, vol. 277, pp. 1445-1451, 1997.

257 [9] M. Roach, C. Marquez, H. S. Yuo, P. Narayan, L. Coleman, U. O. Nseyo, Z. Navvab, and

258 P. R. Carroll, "Predicting the risk of lymph node involvement using the pre-treatment prostate

259 specific antigen and Gleason score in men with clinically localized prostate cancer,”

260 International Journal of Radiation Oncology, Biology, Physics, vol. 28, pp. 33-37, 1994.

261 [10] P. L. Nguyen, M.-H. Chen, K. E. Hoffman, M. S. Katz, and A. V. D’Amico, “Predicting

262 the risk of pelvic node involvement among men with prostate cancer in the contemporary era,”

263 International Journal of Radiation Oncology, Biology, Physics, vol. 74, pp. 104-109, 2009. 
264 [11] L. Cheng, R. Montironi, D. G. Bostwick, A. Lopez-Beltran, and D. M. Berney, "Staging 265 of prostate cancer,” Histopathology, vol. 60, pp. 87-117, 2012.

266 [12] R. Schiavina, V. Scattoni, P. Castellucci, M. Picchio, B. Corti, A. Briganti,

267 A. Franceschelli, F. Sanguedolce, A. Bertaccini, M. Farsad, G. Giovacchini, S. Fanti, W. F.

268 Grigioni, F. Fazio, F. Montorsi, P. Rigatti, and G. Martorana, “11c-choline positron emission 269 tomography/computerized tomography for preoperative lymph-node staging in intermediate-risk 270 and high-risk prostate cancer: comparison with clinical staging nomograms,” European Urology, 271 vol. 54, pp. 392-401, 2008.

272 [13] A. S. Fortuin, W. M. L. L. G. Deserno, H. J. M. Meijer, G. J. Jager, S. Takahashi, O. A.

273 Debats, S. N. Reske, C. Schick, B. J. Krause, I. van Oort, A. J. Witjes, Y. L. Hoogeveen, E. N. J.

274 Th van Lin, and J. O. Barentsz, "Value of PET/CT and MR lymphography in treatment of 275 prostate cancer patients with lymph node metastases,” International Journal of Radiation 276 Oncology, Biology, Physics, vol. 84, pp. 712-718, 2012.

277 [14] M. G. Harisinghani, S. Saini, R. Weissleder, P. F. Hahn, R. K. Yantiss, C. Tempany, B. J. 278 Wood, and P. R. Mueller, “MR lymphangiography using ultrasmall superparamagnetic iron 279 oxide in patients with primary abdominal and pelvic malignancies: radiographic-pathologic 280 correlation,” American Journal of Roentgenology, vol. 172, pp. 1347-1351, 1999.

281 [15] M. G. Harisinghani, J. Barentsz, P. F. Hahn, W. M. Deserno, S. Tabatabaei, 282 C. Hulsbergen van de Kaa, J. de la Rosette, and R. Weissleder, "Noninvasive detection of 
283 clinically occult lymph-node metastases in prostate cancer,” New England Journal of Medicine, 284 vol. 348, pp. 2491-2499, 2003.

285 [16] R. Landry, P. M. Jacobs, R. Davis, M. Shenouda, and W. K. Bolton, "Pharmacokinetic 286 study of ferumoxytol: a new iron replacement therapy in normal subjects and hemodialysis 287 patients,” American Journal of Nephrology, vol. 25, pp. 400-410, 2005.

288 [17] M. Harisinghani, R. W. Ross, A. R. Guimaraes, and R. Weissleder, "Utility of a new 289 bolus-injectable nanoparticle for clinical cancer staging,” Neoplasia, vol. 9, pp. 1160-1165, 2902007.

291 [18] C. U. Herborn, F. M. Vogt, T. C. Lauenstein, O. Dirsch, C. Corot, P. Robert, and S. G. 292 Ruehm, "Magnetic resonance imaging of experimental atherosclerotic plaque: comparison of 293 two ultrasmall superparamagnetic particles of iron oxide,” Journal of Magnetic Resonance 294 Imaging, vol. 24, pp. 388-393, 2006.

295 [19] G. H. Simon, J. von Vopelius-Feldt, Y. Fu, J. Schlegel, G. Pinotek, M. F. Wendland, M.296 H. Chen, and H. E. Daldrup-Link, “Ultrasmall supraparamagnetic iron oxide-enhanced magnetic 297 resonance imaging of antigen-induced arthritis: a comparative study between SHU555C, 298 ferumoxtran-10, and ferumoxytol,” Investigative Radiology, vol. 41, pp. 45-51, 2006.

299 [20] J. J. Pouw, M. Ahmed, B. Anninga, K. Schuurman, S. E. Pinder, M. Van Hemelrijck, 300 Q. A. Pankhurst, M. Douek, and B. Ten Haken, “Comparison of three magnetic nanoparticle 
301 tracers for sentinel lymph node biopsy in an in vivo porcine model," International Journal of

302 Nanomedicine, vol. 10, pp. 1235-1243, 2015.

303 [21] R. Tiguert, E. L. Gheiler, M. V. Tefilli, P. Oskanian, M. Banerjee, D. J. Grignon,

304 W. Sakr, J. E. Pontes, and D. P. Wood, "Lymph node size does not correlate with the presence of 305 prostate cancer metastasis,” Urology, vol. 53, pp. 367-371, 1999.

306

307

308 


\section{Tables}

310 Table 1. Scan parameters for Magnetic Resonance Lymphography.

311

\begin{tabular}{|c|c|c|c|c|c|c|c|c|c|c|}
\hline Name & Description & $\begin{array}{l}\text { Imaging } \\
\text { plane }\end{array}$ & $\begin{array}{l}\text { Echo } \\
\text { time } \\
\text { (ms) } \\
\end{array}$ & $\begin{array}{l}\text { Repetition } \\
\text { time (ms) }\end{array}$ & $\begin{array}{l}\text { Flip } \\
\text { angle } \\
\text { (deg) }\end{array}$ & $\begin{array}{l}\text { Pixel } \\
\text { size } \\
(\mathrm{mm}) \\
\end{array}$ & Matrix & $\begin{array}{l}\text { Slice } \\
\text { thickness } \\
\text { (mm) } \\
\end{array}$ & Slices & $\begin{array}{l}\text { Bandwidth } \\
\text { (Hz/pixel) }\end{array}$ \\
\hline VIBE & $\begin{array}{l}\text { T1-weighted } \\
\text { spin echo }\end{array}$ & coronal & 2.45 & 4.95 & 10 & $\begin{array}{l}0.8 x \\
0.8\end{array}$ & $\begin{array}{l}320 x \\
320\end{array}$ & 0.8 & 240 & 400 \\
\hline MEDIC & $\begin{array}{l}\mathrm{T} 2 *_{-} \\
\text {weighted } \\
\text { gradient echo }\end{array}$ & coronal & 11 & 20 & 10 & $\begin{array}{l}0.8 x \\
0.8\end{array}$ & $\begin{array}{l}320 x \\
320\end{array}$ & 0.8 & 240 & 180 \\
\hline
\end{tabular}

312 


\section{Figure captions}

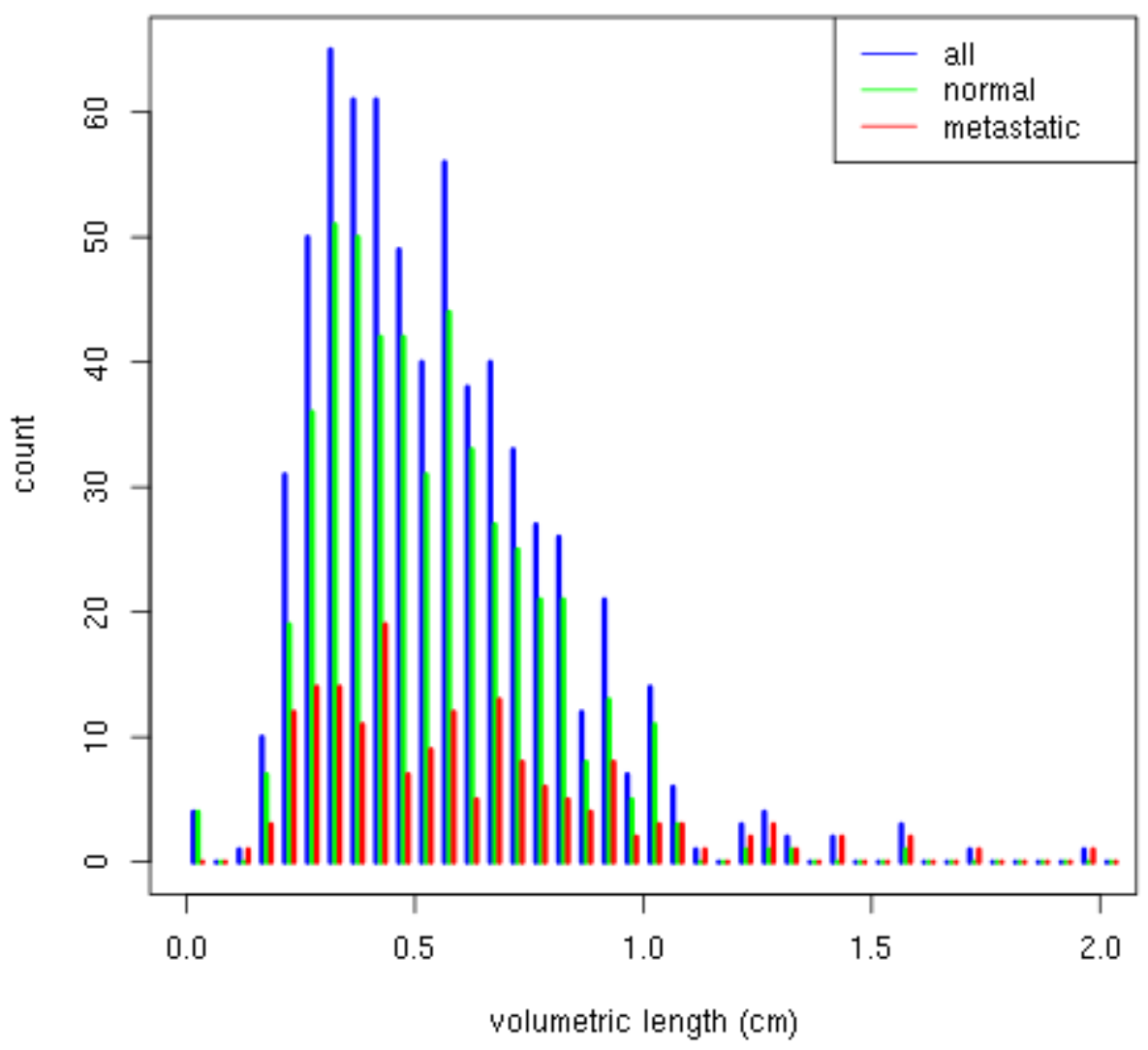

315

316 Figure 1: Histogram of lymph node size. The volumetric length of a lymph node is defined as the 317 cubic root of its volume. 




321 Figure 2: Example of a normal lymph node in ferumoxtran-10 MRL and ferumoxytol MRL.

322 Top row: overviews; bottom row: zoomed-in images. Left pane: USPIO-insensitive 3D T1-

323 weighted (VIBE) sequence. The lymph node is visible as a hypointense structure. Middle pane:

324 3D T2*-weighted (MEDIC) sequence, enhanced with ferumoxtran-10. The normal lymph node

325 is as dark as the fat-suppressed fat and is thus indistinguishable from the background. Right

326 pane: 3D T2*-weighted sequence, enhanced with ferumoxytol. Due to contrast uptake, the

327 normal lymph node is darker than it would have been in non contrast-enhanced MRI, but it is not

328 as dark as the background, and thus may be scored as metastatic. 


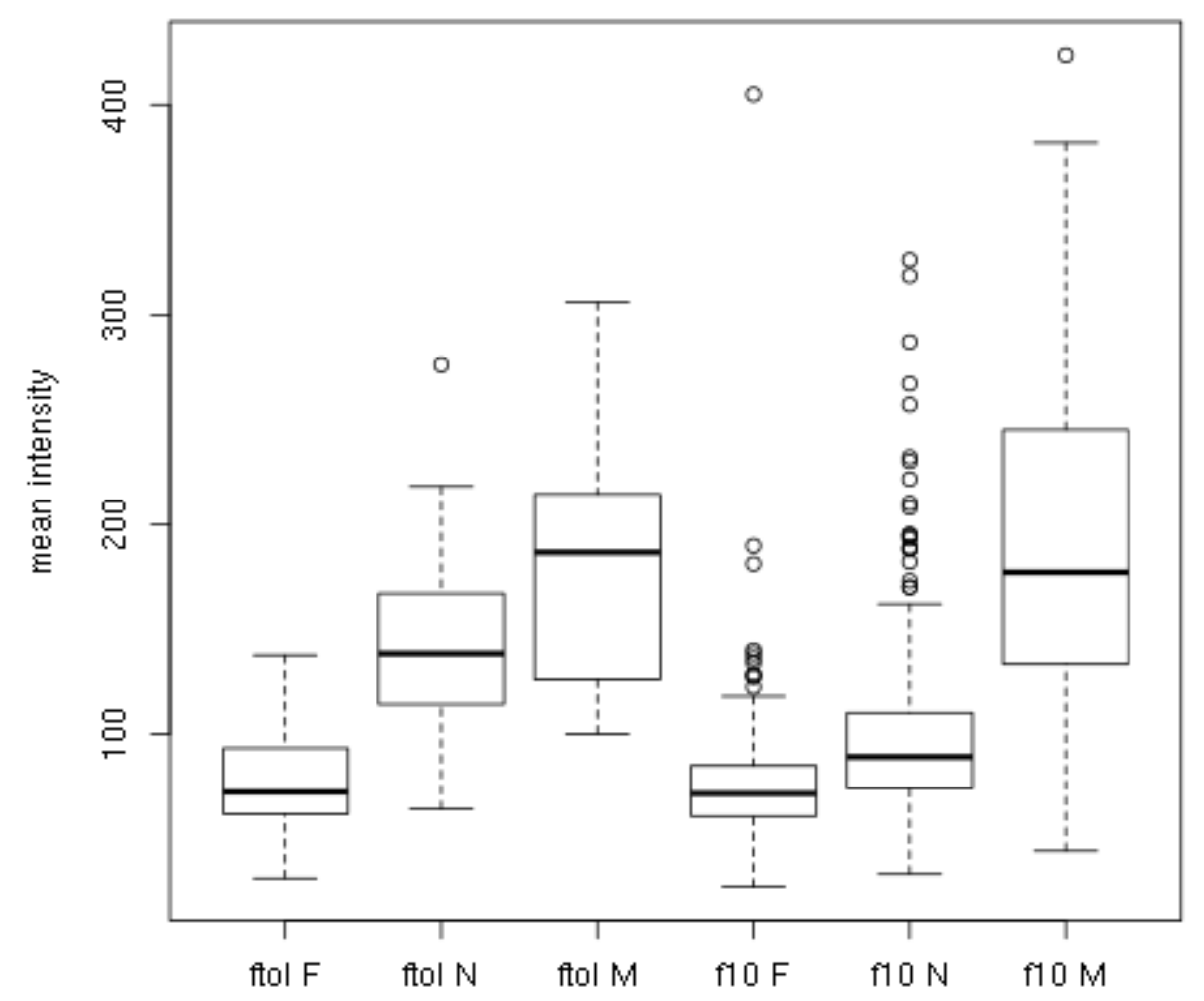

330 Figure 3: Tukey box plot comparing the signal intensities of lymph nodes and fatty tissue regions

331 for both contrast agents.

332 F: fatty tissue regions; N: normal lymph nodes; M: metastatic lymph nodes; ftol: ferumoxytol;

333 f10: ferumoxtran-10. 